\title{
A design rationale for safer terrain park jumps that limit equivalent fall height
}

\author{
Dean Levy ${ }^{1} \cdot$ Mont Hubbard $^{1} \cdot$ James A. McNeil $^{2} \cdot$ Andrew Swedberg $^{3}$
}

Published online: 1 September 2015

(C) The Author(s) 2015. This article is published with open access at Springerlink.com

\begin{abstract}
Ski jump landing surface shapes can be created to cushion jumper landing by specifying a value of equivalent fall height $(\mathrm{EFH})$ but, because the shape is calculated by integrating a differential equation, an infinite number of solutions results from the arbitrary boundary conditions. This paper provides a natural rationale for selection of the least expensive (minimum snow budget) one of these that nevertheless satisfies other design constraints, mainly limited normal acceleration and jerk during approach and landing transitions. Choosing the maximum allowable normal acceleration during the approach transition brings the entire infinite family of landing surfaces as close as possible to the parent slope. Limiting the rate of change of normal acceleration (jerk) decreases the likelihood of loss of balance at takeoff and consequent catastrophic spinal cord injuries on landing. An analogous choice, satisfying limited normal acceleration during the landing transition, selects the single member of the infinite family (providing the desired EFH) that lies closest to the parent slope and is therefore least costly to build. Software in the form of a graphical user interface is described that implements these algorithms and is appropriate for inexperienced users to calculate design details before actual fabrication of landing surfaces at a specific jump site.
\end{abstract}

Mont Hubbard

mhubbard@ucdavis.edu

1 Department of Mechanical and Aerospace Engineering, University of California, Davis, CA 95616, USA

2 Department of Physics, Colorado School of Mines, Golden, CO 80401, USA

3 US Army Maneuver Support Center of Excellence, Fort Leonard Wood, MO 65473, USA

\section{Introduction}

Aerial tricks are now a popular activity for many skiers and snowboarders, and most ski resorts provide dedicated terrain parks jumps allowing enthusiasts to execute these aerial acrobatics. Unfortunately, this has likely contributed to an increase in injuries. Numerous studies have been made concerning this trauma, including those focusing on serious head and spinal cord injuries (SCIs) [1-6]. According to Jackson et al. [5], snow skiing in 2004 replaced football as the second leading cause of SCIs in the US, and these injuries continue [7-12]. Serious SCIs are permanently debilitating and the associated medical and other costs are exorbitant $[13,14]$. Not only is the victim affected for the remainder of his or her life, but often entire families' lives are upended.

Snow parks and affiliates have been reluctant to adopt safer terrain park jump design practices, apparently due both to a questionable risk management strategy and to a lack of understanding of the scientific basis of such design. The current risk management strategy has been to lobby for laws that sharply limit liability, including for negligence, and to require patrons to sign strongly worded waivers thereby placing the burden of safety exclusively on the user. Although owners and operators of terrain parks have been found legally liable for damages from poorly fabricated jumps in the past [15], a more recent court ruling [16] may require a fundamental reassessment of this strategy and the responsibility of resorts for the safety of their patrons.

In this case, the Oregon Supreme Court ruled [16] that due to (a) the inequitable nature of the resort-patron relationship in the formation of the liability waiver contract, and (b) the harsh and inequitable result that would occur if the ski area were released from liability for their own 
negligence, such waivers are procedurally and substantively "unconscionable", respectively, and therefore unenforceable. The court further stated that resorts have a "duty of care" in the creation of snow park jumps because they have "the expertise and opportunity-indeed the common law duty - to foresee and avoid unreasonable risks of their own creation...".

Arguments for avoiding engineering design have been based in part on the belief of the National Ski Areas Association that "standards are impossible" due to rider and snow variability in terrain park jumps [17]. To the contrary, research has shown that it is possible to design and build much safer terrain park jumps [18-26] based on controlling equivalent fall height (EFH), a measure of the energy dissipated in the rider impact at landing and one of the two most important contributing factors to both the likelihood and severity of snow park related injuries. Examples of such jumps have been built and experimentally verified to perform as expected [27].

For these reasons it is essential that resorts develop and implement practices that can demonstrate their "duty of care" while decreasing the number of injuries in general and SCIs in particular. An engineering approach to the design and construction of snow park jumps is perhaps the best way to accomplish this. To this end, the F27 Committee on Snow Skiing of the ASTM International is in the process of developing standards for snow park jumps [28]. The design philosophy discussed here is an attempt to support this process.

To facilitate implementation of safer jump design, we adopt an engineering optimization rationale for choosing a particular solution among the infinite number of solutions $[18,26]$. From the set of EFH-limiting jump landing surfaces, the best is deemed to be the one that minimizes the snow budget (the volume of snow required to build the jump above the parent slope) subject to the physical constraints of the pre-existing parent slope. Snow budget is especially important to terrain park operators because it is a good indicator of total cost in time and resources required to construct a jump. Given a set of designer selected parameters, we show below how choosing to minimize the snow budget selects both the location of the takeoff point and the member of the resulting infinite family of EFHlimited surfaces corresponding to that takeoff point, each of which is closest to the parent slope and thus requires as little extra snow as possible. In some circumstances, such as special events, other criteria such as time in the air may take precedence over snow budget, but as long as the desired criteria can be expressed quantitatively, the basic iterative engineering approach outlined here can be used.

The design is constrained by requiring acceptable maximum normal accelerations of the jumper in both the approach-takeoff and landing transitions. As proposed by
Swedberg [21], the approach-takeoff transition incorporates a classic clothoid shape (used previously in design of roadways and even bobsled-luge tracks [29]). As shown below, the clothoid parameter also makes it possible to limit jerk, the instantaneous rate of change of normal acceleration. The specific landing surface is determined by a similar choice of the landing transition from the EFHlimited surface back to the parent slope that limits normal acceleration.

Even with a proven engineering approach to safer terrain park jumps, the mathematical details are too complex for a person without a scientific background to understand and implement. This motivates the development of automated software in the form of a graphical user interface (GUI) that makes the design and interpretation of impact-limited jumps easy and intuitive for non-technical ski area staff. Finally, we describe an example of software to design these safer snow park jumps that is also applicable to elite athlete training infrastructure. The software is a comprehensive collection of many of the risk-minimizing jump design methods previously described [18-26] and a convenient tool to implement those methods.

\section{Theoretical background}

\subsection{Constant equivalent fall height landing surface}

Figure 1 illustrates the components of a terrain park jump using terminology common in the industry. Two possible landing surfaces are shown. A problem with the typical table top design, which has a generally horizontal deck (table top) and a constant slope landing area, is that impacts are not controlled [22]. Landing just before the knuckle or deep into the landing area can result in unsafe impacts characterized by large EFH. Indeed any straight landing surface will have linearly increasing impact intensity measured by EFH [22]. The present work outlines a design process that avoids these pitfalls in addition to suggesting design criteria that mitigate other risks and corresponding injuries. The most radical change is the use of a constant equivalent fall height landing surface, shown as a solid line in Fig. 1, as an alternative to the (dashed) standard tabletop landing area configuration composed of two straight lines.

The theory of impact-controlled landing surfaces has been discussed in detail by McNeil et al. [26]. We briefly review the theory here for completeness. The equations of motion for the rider center-of-mass while in contact with the approach and landing surfaces are [19]

$\frac{\mathrm{d}^{2} \vec{r}(t)}{\mathrm{d} t^{2}}=-g \hat{y}+(\hat{n}-\mu \hat{v}) \frac{N}{m}-\eta v^{2} \hat{v}$, 


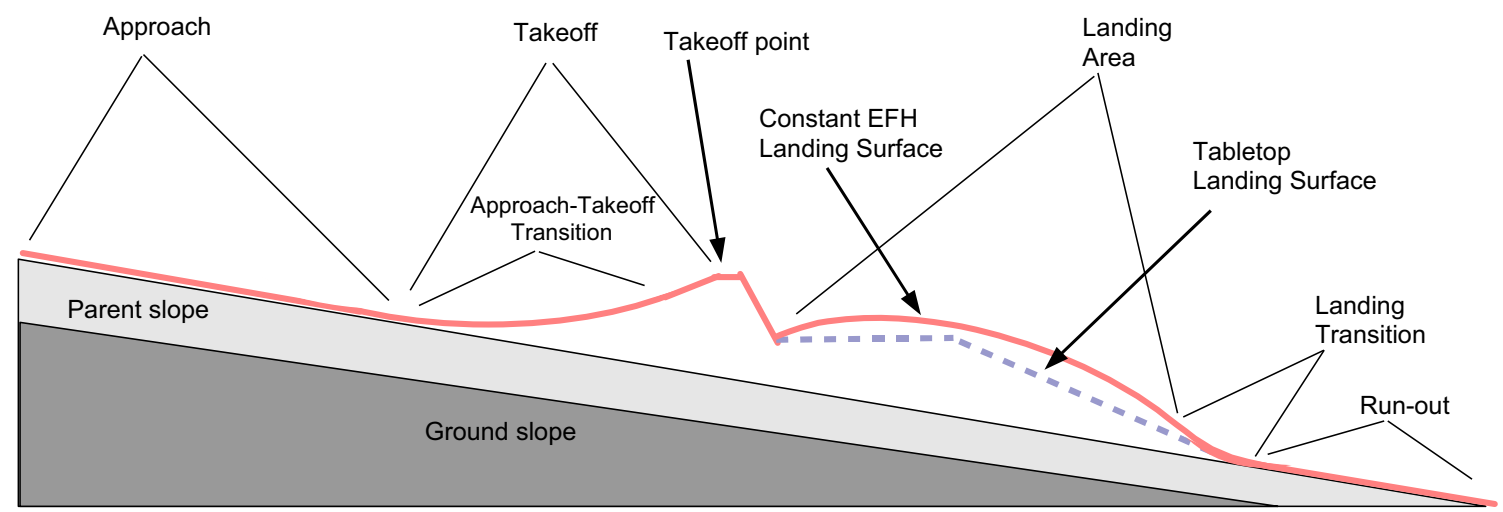

Fig. 1 Components of a standard terrain park jump. Two possible landing surfaces are shown: a standard tabletop landing surface (dashed) and a constant EFH landing surface (solid)

Table 1 Physical parameters

\begin{tabular}{llll}
\hline Parameter & Symbol & Units & Value/range \\
\hline Acceleration of gravity & $\mathrm{g}$ & $\mathrm{m} / \mathrm{s}^{2}$ & 9.81 \\
Mass of jumper & $m$ & $\mathrm{~kg}$ & $60-90$ \\
Air density & $\rho$ & $\mathrm{kg} / \mathrm{m}^{3}$ & $0.85-1.2$ \\
Combined drag parameter & $\eta$ & $\mathrm{m}^{-1}$ & $0.003-0.006$ \\
Coefficient of kinetic friction & $\mu$ & Dimensionless & $0.03-0.12$ \\
Lift to drag ratio & $\rho_{l / d}$ & Dimensionless & $0.0-0.1$ \\
\hline
\end{tabular}

where $g$ is the gravitational acceleration constant, $\vec{r}=(x, y)$ is the (horizontal, vertical) position vector of the rider from the takeoff point suppressing the transverse $(\mathrm{z})$ motion, $\hat{v}$ is the unit velocity vector, $\hat{n}$ is the unit vector normal to the surface, $N$ is the normal force on the rider from the surface, $\mu$ is the kinetic coefficient of friction, and $\eta=\rho C_{d} A_{f} / 2 m$ is the combined (mass specific) drag parameter defined in Eq. 2. The ranges of physical parameters used in this work are given in Table 1 [30-33]. To include wind, the velocity vector in the (last) drag term in Eq. 1 is replaced by the airrider relative velocity, $\vec{v}-\vec{w}$, where $\vec{w}$ is the wind velocity vector. The drag force is given by [31]

$\vec{F}_{d}=-\frac{\rho C_{d} A_{f}}{2} v^{2} \hat{v}=-m \eta v^{2} \hat{v}$

where $A_{f}$ is the frontal area of the rider perpendicular to the direction of travel, $\rho$ is air density, $\vec{v}$ is the velocity, $C_{d}$ is the drag coefficient, and $\eta$ is the combined drag parameter. Hoerner [32] provides approximate values for the rider drag area $C_{d} A_{f}$ for various positions, i.e. standing facing forward $\left(0.836 \mathrm{~m}^{2}\right)$, standing facing sideways $\left(0.557 \mathrm{~m}^{2}\right)$, and tucked facing forward $\left(0.279 \mathrm{~m}^{2}\right)$. Barelle et al. [33] investigate the dependence of skiing drag area on crouch height. Most other discussions [34, 35] of skier drag are from the point of view of how fast elite downhill skiers can go and assume the tuck position.
The magnitude of the normal force is given by [19]

$N=m\left(g \cos \theta_{A}(x)+\kappa(x) v^{2}\right)$,

where $\theta_{A}(x)=-\tan ^{-1}\left(y_{A}^{\prime}\right)$ is the local value of the inclination angle of the hill and $\kappa(x)$ is the local surface curvature,

$\kappa(x)=\frac{y_{A}^{\prime \prime}(x)}{\left(1+y_{A}^{\prime}(x)^{2}\right)^{\frac{3}{2}}}$,

and $y_{A}$ is the approach surface beginning at the start point.

Given any starting point and the approach, approach transition, and takeoff surfaces, the equations of motion, Eq. 1, can be integrated forward to determine the takeoff velocity. The maximum takeoff velocity (resulting from the highest start point and minimum snow friction $\mu$ and air drag $\eta$ ) is called the design speed. This important parameter determines the maximum distance a jumper can cover and thus the overall size of the jump. In practice, where the size of the jump is limited it will be necessary to limit the approach so that the corresponding design speed is not exceeded. As will be discussed further below, conservatism in design is essential. This means that the two most crucial values for parameters are the minima for $\mu$ and $\eta$.

Some care must be taken when estimating the design speed. For single jumps with a well-defined in-run, it is relatively straight-forward to calculate the maximum 
possible takeoff speed using the minimum values for $\mu$ and $\eta$. The inverse of the combined drag parameter, $167<\eta^{-1}<333 \mathrm{~m}$, provides the distance scale over which a rider on a constant slope approaches the terminal speed,

$v_{T}=\sqrt{\frac{g\left(\sin \theta_{A}-\mu \cos \theta_{A}\right)}{\eta}}$,

where $\theta_{A}$ is the (assumed constant) angle of the approach. For jumps without a well-defined start point and where the run-in can be substantially longer than $\eta^{-1}$, the maximum speed before coming to the takeoff can approach the terminal speed for that surface. Similar speeds can be achieved in a jump line following the initial jump. However, the takeoff with its uphill segment and increased friction in the curved transition will reduce this maximum speed by as much as $40 \%$ before reaching the lip. The amount of reduction depends on the specific shape of the takeoff. Where this is known, the maximum takeoff speed should be calculated. Alternatively, in cases where the rider speed could approach the terminal speed prior to the takeoff the US Terrain Park Council recommends adopting a design speed of $80 \% v_{T}$ as a practical guideline.

One of the most important factors affecting the impact safety of a jump is the total energy absorbed on landing. Several authors have proposed the concept of the equivalent fall height $(\mathrm{EFH})$ to characterize this important parameter [18, $19,36]$. Suppose an object falls vertically onto a horizontal surface from a height $h$. Ignoring drag, the speed at impact $v$ is related to the height $h$ through $h=v^{2} / 2 g$. On a sloped landing surface, the impact instead depends principally on the component of the velocity normal to the landing surface $v_{\perp}$, and the relevant energy relation then leads to $h=v_{\perp}^{2} / 2 g$. The component of landing velocity normal to the landing surface is $v_{\perp}=v_{J} \sin \left(\theta_{J}-\theta_{L}\right)$, where $v_{J}$ is the jumper's landing speed, $\theta_{J}$ is the jumper's landing angle, and $\theta_{L}$ is the angle of the landing surface. Thus, the EFH can be expressed as

$h=\frac{v_{J}^{2} \sin ^{2}\left(\theta_{J}-\theta_{L}\right)}{2 g}$

which shows that EFH can be made arbitrarily small by making the angle of the landing surface closely match that of the jumper flight path at landing.

To calculate EFH for an arbitrary jump shape, one must know the shape of the landing surface and solve the equations of motion for the jumper's flight trajectory. The general equations of motion governing center-of-mass flight including lift and drag are [24]

$$
\frac{\mathrm{d}^{2} \vec{r}_{J}(t)}{\mathrm{d} t^{2}}=-g \hat{y}-\eta|\vec{v}-\vec{w}|\left(\vec{v}-\vec{w}-\rho_{l / d} \hat{s} \times(\vec{v}-\vec{w})\right),
$$

where $\rho_{l / d}$ is the lift to drag ratio. Assuming the rider maintains a fixed orientation facing forward, $\hat{s}$ is the unit vector in the "sideways" direction, and the remaining parameters are the same as in Eq. 1. Lift is small in practice $(\sim 1 \%)$ and is neglected here. In general, these equations must be solved numerically but, as shown by McNeil [24], for small to medium-sized jumps (say less than $\sim 12 \mathrm{~m}$ ) the drag can also be ignored at about the $10 \%$ level (with lift effects at the $\sim 1 \%$ level). In an $x y$ coordinate system with origin at the takeoff point and $x$ horizontal, using this approximation allows the classical drag-free closed-form analytic solutions for jumper velocity and position:

$$
\begin{aligned}
& \vec{v}_{J}(t)=\left(v_{J x}, v_{J y}\right)=v_{0} \cos \theta_{T} \hat{x}+\left(v_{0} \sin \theta_{T}-g t\right) \hat{y} \\
& \vec{r}_{J}(t)=\left(x_{J}, y_{J}\right)=v_{0} \cos \theta_{T} t \hat{x}+\left(v_{0} \sin \theta_{T} t-\frac{1}{2} g t^{2}\right) \hat{y},
\end{aligned}
$$

where $\theta_{T}$ is the takeoff angle and $v_{0}$ is initial speed at takeoff ignoring rider "pop" ("pop" refers to the component of skier takeoff velocity perpendicular to the takeoff ramp due to propulsion from the jumping skier's legs). If deemed important, the effect of rider "pop" can be included using the methods of Refs. [23, 24].

From Eq. 8, one can obtain the classic parabolic relation for the jumper flight path $y(x)$

$$
y(x)=x \tan \theta_{T}-\frac{g}{2 v_{0}^{2} \cos ^{2} \theta_{T}} x^{2} .
$$

As shown in Refs. [18, 21, 23], the jumper's landing angle can be found from the two components of the velocity vector in Eq. 7, which can then be used in the definition of $\mathrm{EFH}$ (Eq. 5) to express $h(x)$ in terms of the landing surface shape $y_{L}(x)$

$$
\begin{aligned}
h(x)= & {\left[\frac{x^{2}}{4\left(x \tan \theta_{T}-y_{L}(x)\right) \cos ^{2} \theta_{T}}-y_{L}(x)\right] } \\
& \times \sin ^{2}\left[\tan ^{-1}\left(\frac{2 y_{L}(x)}{x}-\tan \theta_{T}\right)-\tan ^{-1} y_{L}^{\prime}(x)\right],
\end{aligned}
$$

where $\theta_{L}(x)=\tan ^{-1} y_{L}^{\prime}(x)$ has been used.

This expression for EFH characterizes the severity of impact on landing of any jump as a function of the horizontal distance $x$. In fact, once a rider leaves the takeoff with a given initial velocity, his flight path and, in particular, his landing point and EFH are largely determined. When drag and lift are neglected, simple analytic expressions such as Eq. 10 for the EFH can be obtained. It is straightforward however to proceed numerically to determine $h(x)$ including both drag and lift effects.

This relation can also be inverted. By solving for $y_{L}^{\prime}(x)$ from Eq. 10, one obtains a differential equation for the landing surface as a function of a specified $\mathrm{EFH}, h$, 


$$
\begin{aligned}
y_{L}^{\prime}(x)= & \tan \left[\tan ^{-1}\left(\frac{2 y_{L}(x)}{x}-\tan \theta_{T}\right)\right. \\
& \left.+\sin ^{-1} \sqrt{\frac{h}{4\left(x \tan \theta_{T}-y_{L}(x)\right) \cos ^{2} \theta_{T}}-y_{L}(x)}\right] .
\end{aligned}
$$

Equation 11 is the basis of jump landing surface shape design and calculation. Note that $h$ need not be a constant, but a particularly simple and practical jump landing shape can be obtained by taking $h$ to be constant. To find specific instances of constant EFH surface shapes one must solve Eq. 11 by numerical integration. First, we choose values of the parameters $\theta_{T}$ and $h$. Since it is a first order differential equation, one must select a specific boundary condition $y_{L}\left(x_{F}\right)$ at some value of $x_{F}$. For technical reasons [21] related to the behavior of the equation at small values of $x$, it is most convenient to integrate Eq. 11 backward, rather than forward in $x$, so $x_{F}$ is taken to be the terminal point for the constant EFH surface. The arbitrariness of the boundary condition means that there is an infinite number of such solutions for fixed $h$ parameterized by $y_{L}\left(x_{F}\right)$. The freedom to choose $x_{F}$ allows one to address other engineering constraints, such as the snow budget.

The question remains as to what value of the EFH $(h)$ to select. Intuitively, the softer the landing is the safer it is. It has long been recognized that, even in elite ski jumping, large values of EFH are unsafe [36]. However, constant EFH landing surfaces with very low values (e.g. $10 \mathrm{~cm}$ ) will conform to the jumper's trajectory so closely that the rider may not experience the exhilaration of flying which is the principal attraction of the sport. For an upper limit, the US Terrain Park Council [37] recommends that the EFH for any jump landing surface be less than $1.5 \mathrm{~m}$ based on the work of Minetti et al. [38] who found this height to be the maximum that an athletic male could absorb in his leg muscles. The designer is left with the decision. For illustrative purposes, five members of the infinite family of landing surfaces for $\theta_{T}=18^{\circ}$ and $h=1.5 \mathrm{~m}$ are shown in Fig. 2. The boundary conditions for these five landing surfaces were chosen to lie equally spaced along the parent slope, and the one for the lowest lies where the design speed jumper path intersects the parent slope. Before any specific calculations can be made using Eq. 11, however, the location of the takeoff point (the origin of the coordinate system) must be determined.

The takeoff point lies at the end of the approach transition. It is intuitively obvious that, all other things being equal, the closer to the parent slope the takeoff point is, the closer are all the possible resulting safer landing surfaces and therefore the less expensive it will be to construct them. In the next section we first address this question: How close to the parent slope can we put the takeoff point?

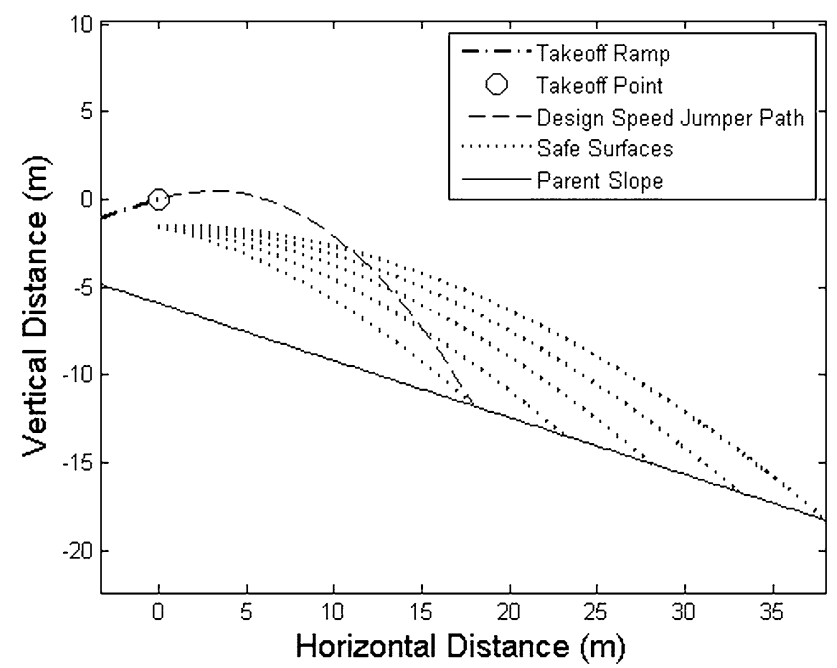

Fig. 2 Five examples from the infinite family of safe landing surfaces yielding EFH $\mathrm{h}=1.5 \mathrm{~m}$ with takeoff angle $\theta_{T}=18^{\circ}$ and parent slope angle $\theta_{A}=18^{\circ}$. The design speed jumper path results from the maximum possible velocity achievable at takeoff $v_{0}=10.2 \mathrm{~m} / \mathrm{s}$, and all other possible jumper flight paths lie below it. To guarantee limited EFH at all speeds up to the design speed, the design speed path must intersect the particular safe landing surface chosen from the infinite family before it intersects the parent slope

\subsection{Approach-takeoff transition}

The role of the approach-takeoff transition is to turn the jumper velocity vector in the vertical plane from down the hill on approach to (typically) upward at takeoff, requiring substantial acceleration perpendicular to the surface path. A "good" transition is here defined as one in which the jumper experiences neither large values of, nor large rates of change of, normal acceleration and thus never feels more than a tolerable amount of slowly changing $\mathrm{g}$ forces. Large normal accelerations are undesirable for obvious reasons, but so are large rates of change of these accelerations (jerk) because they can cause the jumper to lose balance. Disequilibrium is especially dangerous just before takeoff because it can result in unwanted inverting rotation during flight increasing the likelihood for serious injury such as SCI. This is a similar approach to that used by the International Ski Federation (FIS) in the design of competition approach transitions for elite alpine jumpers [39]. FIS standards are quite conservative, allowing only about $0.7 \mathrm{~g}$ of normal acceleration [39]. We have assumed below that a non-elite skier or snowboarder can reasonably tolerate about $1.5 \mathrm{~g}$.

We assume here that the best approach transition curve minimizes the snow budget required for its fabrication (as well as that of the resulting infinite family of safe landing surfaces by keeping them close to the parent slope) while smoothly guiding the jumper from the approach to the 
takeoff. We will show below that this minimum snow budget transition will be one which is at the limiting tolerable values of acceleration and jerk, $a_{\max }$ and $j_{\max }$, respectively. That is, a transition exhibiting maximum acceleration or jerk less than the maximum permitted always requires more snow to build.

A naive first choice for the transition curve might be a circular segment. A purely circular portion, with a radius chosen to produce the acceleration limit, provides the quickest turning transition and the least deviation of the takeoff point upward away from the parent slope. But this subjects the jumper to instantaneous changes in normal acceleration at the entrance to and exit from the circular portion. A clothoid transition curve (also called the Euler spiral) gradually changes jumper normal acceleration and has been proposed for the ski jump application [21].

The clothoid has the unique property of a linearly decreasing radius of curvature with increasing arc distance. It satisfies the equation:

$A^{2}=s r$,

where the clothoid parameter A, called the spiral flatness, is a constant length to be determined below, $s$ is arc length along the clothoid curve, and $r$ is the radius of curvature at arc length $s$. International Ski Federation design rules for large Nordic jumping hills used in elite Olympic and World Cup competitions [39] contain a similar requirement for a "clothoid-like [approach] transition curve". Unfortunately, a purely clothoidal transition is not optimal because it does not minimize the snow budget.

To provide faster turning while also meeting both normal acceleration and jerk requirements, we include a circular segment between two mirror-image clothoid segments. These entry and exit clothoids smoothly vary the curvature (and thus the normal acceleration) from zero up to a maximum at the beginning of the circular segment and back again to zero at the beginning of the straight takeoff ramp. The relative values of the limits on jerk and acceleration then determine the fractions of the total turning angle provided by the respective component curves as described below. We assume the clothoid and circular segments are symmetric about the center of the transition. Thus, the total transition shape is known if half the transition is defined. Let $\xi$ be half the total transition turning angle:

$\xi=\frac{\theta_{A}+\theta_{T}}{2}$

where $\theta_{A}$ is the approach (parent) slope angle and $\theta_{T}$ is the takeoff angle. Next, we define the parameter $\gamma$ as the fraction $(0 \leq \gamma \leq 1)$ of the total turning angle subtended by the circular section. In other words, the larger $\gamma$ is, the more completely circular the transition is. Therefore, the remaining portion of the angle turned by each clothoid segment is:

$\xi_{c}=(1-\gamma) \frac{\theta_{A}+\theta_{T}}{2}$

To determine the approach-takeoff transition we first impose the normal acceleration constraint. Normal jumper acceleration is inversely proportional to the radius of curvature at the point of interest. The velocity is approximated to be constant along the transition. This is a valid assumption only if the transition length is short compared to the length of the entire approach section, which is probably true for most terrain park jumps. Thus the maximum tolerable acceleration determines the maximum curvature (minimum radius) of the clothoid,

$r_{\min }=\frac{v^{2}}{a_{\max }}$

where $r_{\text {min }}$ is the minimum radius, $v$ is the speed at the entrance to the clothoid, and $a_{\max }$ is the maximum tolerable radial acceleration in the transition. We assume that the central circular segment is position and slope continuous with the clothoid, and that the radius of the circular segment and the minimum radius of the clothoid are equal.

When all other quantities (e.g. the design speed and the jerk constraint) are held constant, the effect of the acceleration constraint $a_{\max }$ is fairly straightforward. The more acceleration $a_{\max }$ is permitted, the smaller the minimum (circular) radius $r_{\min }$ will be and the closer both the entrance to the straight takeoff ramp and the takeoff point will be to the parent slope.

Next we impose the normal jerk constraint. Jerk is defined as the time derivative of the acceleration; thus one finds an expression for the normal component of the jerk $j_{\perp}$,

$j_{\perp}=\frac{\mathrm{d} a_{\perp}}{\mathrm{d} t}=\frac{\mathrm{d} a_{\perp}}{\mathrm{d} s} \frac{\mathrm{d} s}{\mathrm{~d} t}$

$=\frac{v^{3}}{A^{2}}$

where we have used the two relations

$$
\begin{gathered}
v=\quad \frac{\mathrm{d} s}{\mathrm{~d} t} \\
a_{\perp}=\frac{v^{2}}{r}=\frac{s v^{2}}{A^{2}}
\end{gathered}
$$

Note that if velocity is roughly constant, as we have assumed above, then Eq. 17 implies that the normal jerk $j_{\perp}$ is also roughly constant. The jerk is used to determine $\gamma$ through a differential analysis of turning on a clothoid [21]. The differential arc length of a clothoid is proportional to its differential turning angle through the radius of curvature. 
$\mathrm{d} s=r \mathrm{~d} \theta=\frac{A^{2}}{s} \mathrm{~d} \theta$

Thus, integrating Eq. 18 and using Eq. 12 evaluated at the end of the entrance clothoid yields:

$$
\begin{gathered}
\int_{s_{i}=0}^{s_{f}} s \mathrm{~d} s=\int_{\theta_{i}=0}^{\theta_{f}} A^{2} \mathrm{~d} \theta \\
\frac{s_{f}^{2}}{2}=A^{2} \xi_{c}
\end{gathered}
$$

where $s_{f}$ is the final arc length of the clothoid. From the clothoid definition the final arc length must be inversely proportional to the minimum radius.

$s_{f}=\frac{A^{2}}{r_{\min }}$

Substituting for $s_{f}$ and $\xi_{c}$, yields an expression for $\gamma$ in terms of $A, \theta_{A}$, and $\theta_{T}$.

$\gamma=1-\frac{A^{2}}{r_{\min }^{2}\left(\theta_{A}+\theta_{T}\right)}$

The rationale for limiting jerk may be clear, but the appropriate limiting value for terrain park jumps is not. Nevertheless the jerk limit is important since it insures not only that the accelerations during transition are tolerable, but that they are also smoothly, and not suddenly, applied.

Some numerical examples illustrating the effect of the jerk constraint $j_{\max }$ are compared in Fig. 3. Shown are four possible transitions emanating from the same initial point on a parent slope with angle $\theta_{A}=18^{\circ}$, takeoff ramp angle $\theta_{T}=18^{\circ}$, jumper design speed $v_{0}=10.15 \mathrm{~m} / \mathrm{s}$ and the speed just before the transition $v=13.67 \mathrm{~m} / \mathrm{s}$ and with constraint on the maximum acceleration $a_{\max }=1.5 \mathrm{~g}$, and corresponding $r_{\min }=12.7 \mathrm{~m}$. The four transitions are for varying circular turning fractions $(\gamma=1.0,0.58,0.37$, and 0 ) and corresponding $j_{\max }=\inf , 6,4$, and $2.5 \mathrm{~g} / \mathrm{s}$. The topmost transition corresponds to all turning on the circular segment $(\gamma=1.0)$, has infinite jerk at its entrance and exit, and contains no clothoidal segments at all. The remaining three show the effect of limiting jerk to smaller and smaller values. The last of these has jerk limited enough that all the required turning can be accomplished on the clothoid segments alone, and no circular segment is required. For even lower jerk limits, all required turning can be achieved on a purely clothoidal transition and before reaching the acceleration limit. In general, as the jerk limit decreases the takeoff point lies further from the parent surface.

Normal acceleration versus distance is seen in Fig. 4, for the same four transitions shown in Fig. 3. When normal jerk is limited to $j_{\max }$, the resulting approach transition exhibits a gradual (nearly linear) increase in normal acceleration along the entrance clothoid; a relatively steady

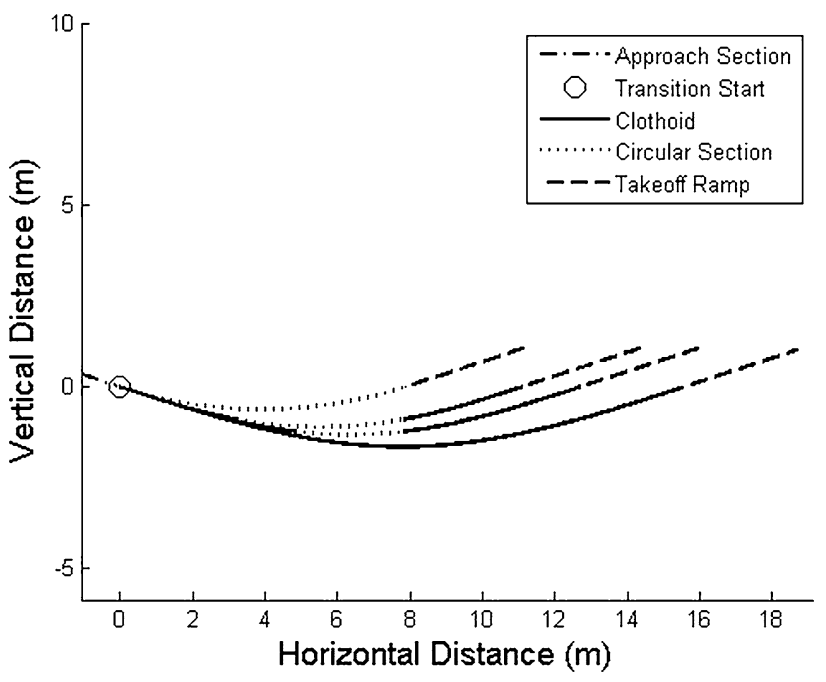

Fig. 3 Four possible approach transitions from the same point on a parent slope with angle $\theta_{A}=18^{\circ}$ to takeoff ramp angle $\theta_{T}=18^{\circ}$, constraint on maximum acceleration $a_{\max }=1.5 \mathrm{~g}$, design speed $v_{0}=$ $10.2 \mathrm{~m} / \mathrm{s}$ and velocity at the beginning of the transition $v=13.7 \mathrm{~m} / \mathrm{s}$. The four transitions have varying jerk weighting $\left(j_{\max }=\inf , 6,4\right.$, and $2.5 \mathrm{~g} / \mathrm{s}$ ) and corresponding circular turning percentages $(\gamma=1.0,0.58,0.37$, and 0$)$. The top transition has all turning on the circular segment $(\gamma=1.0)$, the second two have circles sandwiched between clothoid segments, and the last is entirely composed of two clothoids. All four contain the same straight takeoff ramp at the end. As maximum allowable jerk is decreased, the end of the transition lies further from the parent slope, the jump requires more snow, and it is more expensive to build

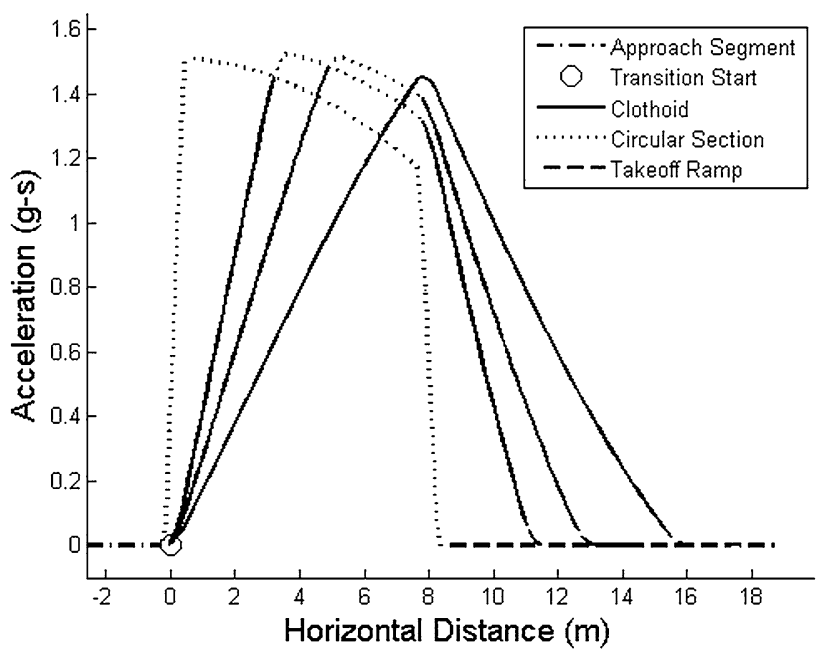

Fig. 4 Magnitude of normal acceleration versus distance for the four approach transitions shown in Fig. 3. The purely circular transition has sudden changes in acceleration. As the maximum jerk constraint decreases, the transitions require more distance and more snow to build

normal acceleration throughout the circular segment (changing only because of small changes in speed due to drag, snow friction and elevation changes); and a gradual 
decrease in normal acceleration to zero along the exit clothoid. The slight decreases in normal acceleration along the circular segments are due to small changes in velocity. As the maximum value of allowable jerk is decreased, more and more transition length is required to turn the velocity vector, and the end of the transition lies further and further from the parent slope, as is obvious from Fig. 3. Limiting jerk is costly in the same sense that limiting acceleration is: more snow is required to build a jump with more severely limited jerk and/or acceleration.

Note also that all transitions shown in Fig. 3 contain a takeoff ramp that is exactly straight and long enough to give the jumper time to recover from the substantial normal accelerations felt during the transition $[25,26]$. The US Terrain Park Council (USTPC) has proposed [37] that the straight section of the takeoff be at least the nominal design takeoff speed times 1.5 human reaction times, or about 0.3 s. A comparative requirement (using a time of $0.25 \mathrm{~s}$ ) is contained in the design rules provided by FIS for large Nordic jumping hills used in elite Olympic and World Cup competitions [39].

If we are given the four specified parameters $j_{\max }, a_{\max }$ and the approach and takeoff angles $\theta_{A}$ and $\theta_{T}$ the design procedure would proceed as follows: speed $v$ at the beginning of the approach transition would be determined by integrating Eq. 1 along the parent slope from the maximum height starting point to the beginning of the approach transition. The minimum radius $r_{\text {min }}$ would be calculated from Eq. 15, $v$, and the specified acceleration limit $a_{\max }$. The spiral flatness $A$ would then be determined from Eq. 17, $A$, and $j_{\max }$. Finally $\gamma$ could be calculated from Eq. 20.

Because no empirical studies have been done on the effect of jerk on rider balance and thus no guidelines exist in the literature for tolerable values of jerk $j_{\max }$, in the software we proceed in an alternate way. We instead arbitrarily choose constant $\gamma=0.5$ and rely on the other three parameters $a_{\max }, \theta_{A}$ and $\theta_{T}$ (we believe this choice provides a reasonable tradeoff between jumper safety and snow budget but more research is needed in this area). The design algorithm implemented is as follows: find $v$ and $r_{\min }$ as above but in this case when the percent circular turning $\gamma$ is specified rather than $j_{\max }$, then Eq. 20 (rather than Eq. 17) is used to determine the clothoid parameter $A$.

\subsection{Landing transition}

Recall from Section $2 \mathrm{~A}$ that the requirement that the landing surfaces provide a specified EFH yields an infinite set of solutions $[18,26]$. Thus far, the approach transition has been made as compact as possible while satisfying design constraints on maximum normal acceleration and jerk. As a result the takeoff point and the critical point (a singular point through which every member of the infinite family of constant EFH landing surfaces passes [21]) is as close as possible to the parent slope. In this sense all the members of infinite family are as close as possible to the parent slope (and require as little extra snow as possible to construct, whichever one of them is eventually chosen). We now explain how to choose the single member of this family that is closest to the parent slope and least expensive of all.

This final phase in the design process proceeds by focusing on the impact point of the design speed jumper path (the outermost of all possible jumper paths), which crosses every surface in the infinite family (Fig. 5). This impact point marks the end of the constant EFH landing surface and the beginning of the landing transition from the constant EFH surface back to the parent slope. The requirements of the landing transition are similar to those of the approach transition, but less severe: (1) the surface must be point and slope continuous with the constant EFH landing surface prior to the transition; (2) the jumper must not experience too large a normal acceleration (for typical jumps, $3 \mathrm{~g}$ is probably too large); and (3) the landing transition must minimize the snow budget, without violating the maximum acceleration limitation. Less care need be taken with limiting normal acceleration in the landing transition because the effects of possible jumper loss of control of orientation are less potentially catastrophic. Flight and landing have already occurred with controlled

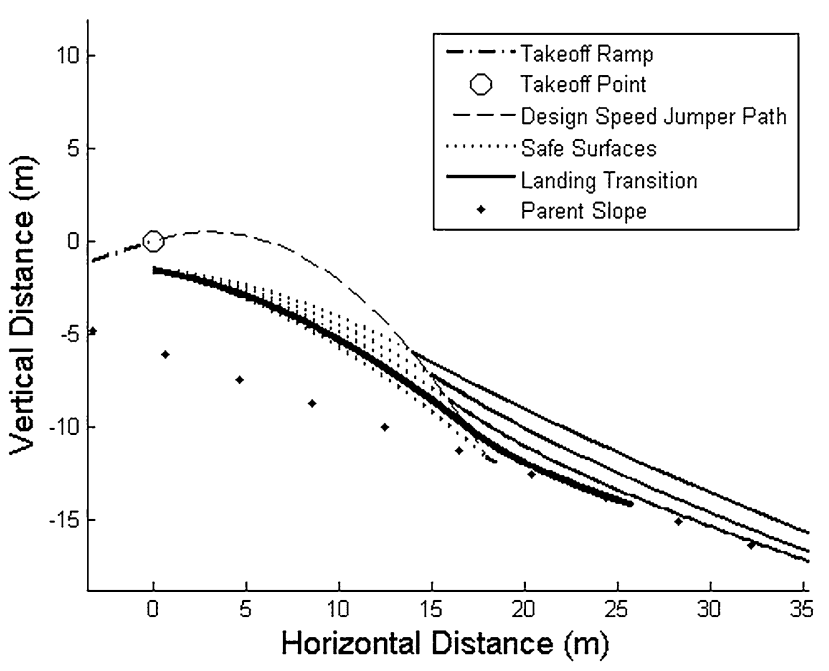

Fig. 5 The design speed jumper flight path (dashed) crosses the entire infinite family (small dots) of constant EFH landing surfaces with the desired EFH. The design method chooses the member of the infinite family closest (dark solid) to the parent slope (large dots) that still allows an exponential landing transition curve with slope and point continuity at the impact point with the design speed jumper path and a limited normal acceleration during sliding after impact. This landing surface and transition is the least expensive to build because it is closest to the parent slope and thus has the lowest snow budget 
impact. Nevertheless, a fall after landing due to jumper inability to handle large normal accelerations should be avoided.

The landing transition shape (the vertical height difference between the transition snow surface and the parent slope) is modeled as an exponential of the form,

$y_{E}(x)=C e^{-\frac{x-x_{0}}{a}}$

where $y_{E}(x)$ (when $x>x_{0}$ ) is the transition's vertical distance from the parent slope, $C$ is a constant to be determined, $x$ is the horizontal distance coordinate and the independent variable, $x_{0}$ (to be chosen) is the $\mathrm{x}$ coordinate at the beginning of the transition, and $a$ is the characteristic length of the exponential. This form of the landing transition shape is simpler than that for the clothoid-based approach-takeoff transition and is sufficient because the adverse consequences of loss of balance are less likely to be severe on landing than at takeoff. The decision to impose acceleration limitations on the transition ultimately winnows the infinite family of constant EFH landing surfaces to a single surface.

Determining the shape of the landing transition function $y_{E}(x)$ and the point $x_{0}$ where it begins is dependent on the maximum normal acceleration permitted on the transition. This occurs, in general, where the constant EFH landing surface and landing transition meet. Recall that the normal acceleration is the product of the curvature of the surface and the square of the velocity at any point. Because the transition is modeled as a decaying exponential, the point on the transition where it meets the constant EFH surface has the greatest curvature. We also assume that the jumper sliding velocity is greatest at the beginning of the transition. The steeper the landing transition's initial slope (at $x_{0}$ ), the shorter the distance over which the transition curve settles. In other words, the characteristic distance $a$ is determined from and inversely proportional to the initial landing transition slope. The smaller the characteristic distance $a$, the shorter the distance over which the transition occurs back to the parent slope.

As seen in Fig. 5 the magnitude of the slope of the landing transition curve, once it is chosen, decreases as the distance from the takeoff point increases. Since the landing transition and constant EFH surface curves are slope continuous, the best landing transition is the one that begins farthest from the takeoff point because it has the smallest snow budget. In Fig. 5, the total area under the constant EFH surface decreases as the transition point is moved farther from the takeoff point because the particular constant EFH surface from the infinite family is also closer to the parent slope. Therefore the desired transition point occurs at the largest horizontal distance $x_{0}$ along the design speed jumper path from the takeoff point for which the maximum acceleration constraint during the ensuing transition is still satisfied. We calculate the parameter $x_{0}$ with the following iterative algorithm, which essentially moves along the design speed jumper path continually testing whether the corresponding transition, back to the parent slope from the constant EFH landing surface passing through that point, has the limiting value of normal acceleration.

- Integrate the design speed jumper flight path forward from takeoff.

- Guess the coordinate $x_{0}$ of the point on the path where the landing transition might begin.

- Evaluate the transition function at $x_{0}$ and solve for the constant $C$

$y_{E}\left(x_{0}\right)=C$,

where $C$ is the vertical distance between the present location on the design speed jumper path (at the start of the transition) and the parent slope. This ensures that the constant EFH landing surface and transition surface are position continuous.

- Determine the derivative $y_{E}^{\prime}\left(x_{0}\right)$ of the constant EFH landing surface at $x_{0}$ from Eq. 11 .

- Slope continuity requires that the derivative of the constant EFH landing surface must equal the sum of the derivative of the transition surface at this point and the derivative of the parent slope, or

$\frac{\mathrm{d} y_{E}}{\mathrm{~d} x}\left(x_{0}\right)=y_{E}^{\prime}\left(x_{0}\right)-\tan \theta_{P S}$,

where $\theta_{P S}$ is the angle of the parent slope.

- Use the expression for the derivative of the transition function evaluated at $x_{0}$ to solve for $a$ :

$\frac{\mathrm{d} y_{E}}{\mathrm{~d} x}\left(x_{0}\right)=-\frac{C}{a} \rightarrow a=-\frac{C}{\frac{\mathrm{d} y_{E}}{\mathrm{~d} x}\left(x_{0}\right)}$.

- Determine the normal acceleration of the rider at the beginning of the exponential transition curve

$a_{\perp}=\kappa\left(x_{0}\right) v^{2}$,

where $a_{\perp}$ is the normal acceleration, $\kappa$ is the curvature (Eq. 4) of the transition, and $v$ is jumper velocity.

- If the acceleration $a_{\perp}$ is less than allowable, go back to the second step and increase the coordinate $x_{0}$, else terminate and the transition begins at the present value of $x_{0}$. 


\section{Numerical software implementation}

The rationale described in Sect. 2 chooses the single least expensive member from the infinity of solutions that has the specified impact performance $\mathrm{EFH}=h$ at a chosen takeoff angle $\theta_{T}$, while also possessing low normal acceleration transitions from the parent slope to the takeoff ramp and from the constant EFH landing surface back again to the parent slope. The steps required are somewhat intricate and the computations are not well suited to hand calculation. For this reason, using MATLAB we have created numerical software with a GUI to facilitate implementation of this procedure by the designer. The GUI consists of a single window with two main panes (Fig. 6). The left pane allows easy specification of the values of input variables using sliders and text boxes, while the right pane displays the subsequent computations graphically. Control of program flow is achieved with buttons at the top. Input variables are separated into slope variables: (parent slope inclination $\theta_{P S}$ and locations of the starting point and the jump on the terrain), and jump variables (or design choices): EFH $h$ and takeoff angle $\theta_{T}$ and the option to include jumper pop in the calculations or not.

The software was designed primarily as a tool for users with little or no engineering or technical scientific background. For this reason, it is programmed to limit the potential for unrealistic jump designs or other abuses of the design method by systematically guiding the user through the design process and limiting all user-entered parameters to commonly accepted, reasonable ranges. The interaction between user and program is established in a message board (top, left of Fig. 6) which instructs the user to enter parameters, gives warnings for missing parameters, and provides useful jump-related output data.

All quantities internal to the software are listed in Table 2. Variables, such as the tolerable acceleration and $\gamma$, come pre-set within the program and are not changeable by the user. One paramount safety concern in jump design is to ensure that no jumper overshoots the intended landing area. Several parameters, including the minimum snow coefficient of friction $\mu=0.03$ [30] and drag parameter $\eta$ $[32,33]$, are not left to the discretion of the user either. Cautious (low) values for these two parameters result in a similarly conservatively high design speed, guaranteeing that the length of the jump calculated is sufficient to accommodate all reasonable jumper speeds and thereby to prevent over-jumping.

It is important to give the designer creative latitude however. Once they have been guided through the first jump design, they are free to engage the program further in an iterative design process. After entering exogenous variables including the parent slope angle, jumper starting point, and location of the approach section, as well as the jump design variables (required EFH and takeoff angle), the program displays the complete profile of the designed constant EFH jump, from the jumper starting position to the end of the landing transition, as well as key jumper and design information including snow budget, maximum takeoff (design) speed, maximum jump distance, maximum jump height above the constant EFH landing surface, and

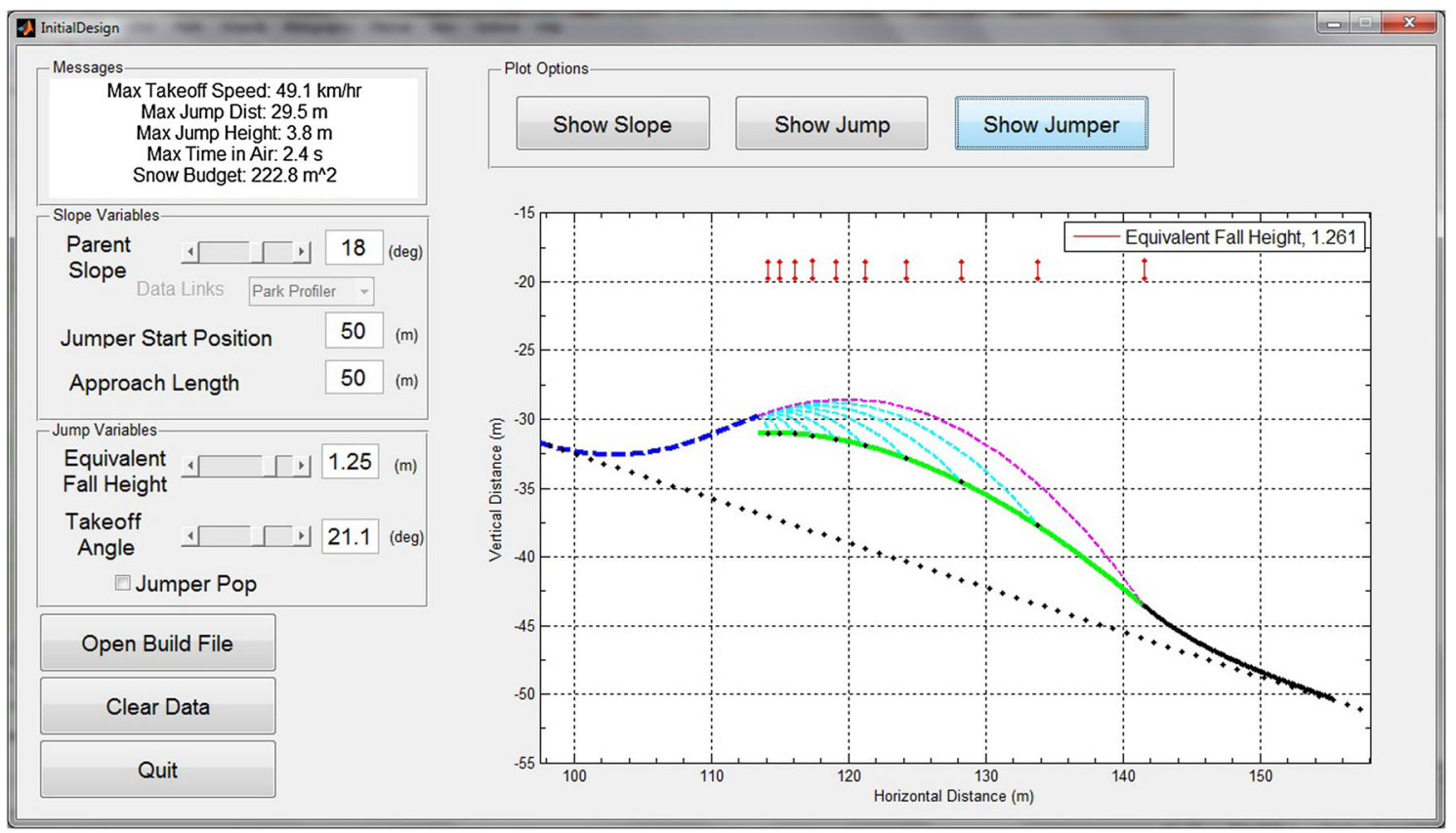

Fig. 6 Graphical user interface for constant EFH landing surface design 
Table 2 Default internal software parameters

\begin{tabular}{llll}
\hline Parameter & Symbol & Units & Value/range \\
\hline Maximum approach transition g-forces & $a_{\max }^{A}$ & $\mathrm{~g}$ & 1.5 \\
Maximum landing transition g-forces & $a_{\max }^{L}$ & $\mathrm{~g}$ & 3.0 \\
Percent circular turning & $\gamma$ & Dimensionless & 0.5 \\
Pop velocity & $v_{P}$ & $\mathrm{~m} / \mathrm{s}$ & 1.2 \\
Drag area & $C_{d} A_{f}$ & $\mathrm{~m}^{2}$ & 0.279 \\
Coefficient of kinetic friction & $\mu$ & Dimensionless & 0.03 \\
Air density & $\rho$ & $\mathrm{kg} / \mathrm{m}^{3}$ & 0.85 \\
Mass of jumper & $m$ & $\mathrm{~kg}$ & 75 \\
\hline
\end{tabular}

maximum air time (shown at top left of Fig. 6). The graphical output presents a close-up view of the jump (Fig. 6) along with several feasible jumper trajectories and corresponding values of EFH. If the designer is satisfied with the displayed jump they may print out instructions to build it along with the parameter values used; otherwise, all or some parameters may be adjusted until the design is finalized.

Ultimately, the most important output of the program is the shape of the entire jump. This is provided not only graphically as shown in Fig. 6, but also optionally as a Build File, a text file containing a list of $x y$ coordinates specifying the shape of the whole jump including the approach-transition and takeoff, the jump landing surface itself, and the landing transition. These are presented as vertical distances from the parent slope as a function of distance along the slope and are essential in actually building the proposed design [27].

\section{Discussion}

Although the concept of minimum landing impact design in general and the software implementation in particular have been here proposed for terrain parks at ski resorts used by the public, they are not limited to that application. In a recent Austrian study of World Cup elite skiers [40], the concept of EFH has already been extended (to equivalent landing height, ELH) to account for the fact that landing occurs over a finite time and that therefore landing surface curvature can also be important. Furthermore, nearly half the US Ski Team athletes are injured in training each year, many from impacts suffered in jumping, which has motivated interest from the US Ski and Snowboard Association in safer design of elite athlete training facilities utilizing these concepts [41]. This problem of safety in skiing is now a pressing international one, and it has motivated a recent meeting at International Olympic Committee Headquarters to review the current knowledge on freestyle skiing and snowboarding injury risk, etiology and mechanisms, and needed research in the area and to propose practical injury prevention measures in these events in the future [42]. We believe the ideas presented in the present paper have a great deal to add toward increasing safety in all forms of skiing that include flight and landing impact.

In addition, in the United States the ASTM F27 Snow Skiing Committee is in the early stages of developing terrain park jump standards. These efforts will require a greater reliance on engineering methods and motivate the creation and adoption of intuitive design tools such as the constant EFH jump design software presented here. This software has already been used to design an exemplar jump before then fabricating and testing it to compare its actual performance with the design expectations, thereby experimentally validating the efficacy of this approach [27, 28].

The present rationale relies on a design speed resulting from a well-defined start point. Rather than isolated jumps, however, it has become more common in terrain parks to use closely-spaced series of jumps, called a "jump line". In this case, the terminal point for one jump (and the corresponding velocity there) would become the start point for the next jump and used to determine its design speed. This conservative approach in the software produces more robust jump designs that are able to tolerate the inevitable variability in design parameters, ultimately making for even safer jumps.

Throughout we have implicitly assumed jumps made entirely from snow. But there is no reason in principle that the supporting jump surface shape could not be crafted permanently from soil in the summer, with a somewhat thinner snow layer added and maintained each winter from either man-made or natural snow. This might make sense in areas with little or no natural snow.

\section{Summary}

A design rationale has been presented for terrain park jumps at ski resorts available to the general public. In addition to straight takeoff ramps which decrease the 
possibility of inadvertent inversion during flight, these jumps include landing surfaces shaped to limit the EFH on landing, thereby decreasing the likelihood and severity of injury due to landing impact. Suitable approach and landing transition shapes have been chosen so that the resulting jump has the minimum snow budget required for fabrication while limiting maximum normal accelerations and jerk and exactly limiting jumper landing impact (EFH) for all possible jumper speeds (below the design speed). Restricting acceleration and jerk during the approach transition decreases the possibility of loss of balance on takeoff and consequent flight rotations that result in back, head and neck landings. Example software has been described which implements these considerations in an organized and graphical way, appropriate for any user with reasonable practical knowledge of snow jumps but not requiring detailed scientific or engineering expertise. We intend to promulgate this design philosophy by implementing the design rationale in other more common computational environments including Excel.

Open Access This article is distributed under the terms of the Creative Commons Attribution 4.0 International License (http://crea tivecommons.org/licenses/by/4.0/), which permits unrestricted use, distribution, and reproduction in any medium, provided you give appropriate credit to the original author(s) and the source, provide a link to the Creative Commons license, and indicate if changes were made.

\section{References}

1. Tarazi F, Dvorak MFS, Wing PC (1999) Spinal injuries in skiers and snowboarders. Am J Sports Med 27:2

2. Meyers AR, Misra B (1999) Alpine skiing and spinal cord injuries: view from a national database. In: Johnson RJ (ed) Skiing trauma and safety: 12th volume, STP 1345. ASTM International, West Conshohocken, PA, pp 150-157

3. Yamakawa H, Murase S, Sakai H, Iwama T, Katada M, Niikawa S, Sumi Y, Nishimura Y, Sakai N (2001) Spinal injuries in snowbarders: risk of jumping as an integral part of snowboarding. J Trauma 50:1101-1105

4. Seino H, Kawaguchi S, Sekine M, Murakami T, Yamashita T (2001) Traumatic paraplegia in snowboarders. Spine 26(11):1294-1297

5. Jackson AB, Dijkers M, DeVivo MJ, Poczatek RB (2004) A demographic profile of new traumatic spinal cord injuries. Arch Phys Med Rehab 85:1740-1748

6. Ackery A, Hagel BE, Provvidenza C, Tator CH (2007) An international review of head and spinal cord injuries in alpine skiing and snowboarding. Inj Prev 13:368-375

7. Greve MW, Young DJ, Goss AL, Degutis LC (2009) Skiing and snowboarding head injuries in 2 areas of the United States. Wilderness Environ Med 20:234-238

8. Moffat C, McIntosh S, Bringhurst J, Danenhauer K, Gilmore N, Hopkins CL (2009) Terrain park injuries. Western J Emerg Med 10, 4

9. Brooks MA, Evans MD, Rivara FP (2010) Evaluation of skiing and snowboard injuries sustained in terrain parks versus traditional slopes. Inj Prev 16:119-122

10. Henrie M, Petron D, Chen Q, Powell A, Shaskey D, Willick S (2011) Comparison of ski and snowboarding injuries that occur inside versus outside terrain parks. Presentation at international society for skiing safety congress, Keystone, CO, May 1-7

11. Russell K, Meeuwisse W, Nettel-Aguirre A, Emery CA, Wishart J, Rowe BH, Goulet C, Hagel BE (2011) Snowboarding injury rates and characteristics sustained on terrain park features. Presentation at international society for skiing safety congress, Keystone, CO, May 1-7

12. Russell K (2011) The relationship between injuries and terrain park feature use among snowboarders in Alberta, $\mathrm{Ph}$. D. thesis, University of Calgary, Alberta, Canada

13. Dijkers M, Abela M, Gans B, Gordon W (1995) The aftermath of spinal cord injury. In: Stover SL, DeLisa JA, Whiteneck GG (eds) Spinal cord injury: clinical outcomes from the model systems. Aspen Publishers, Gaithersburg, pp 185-212

14. DeVivo M, Whiteneck G, Charles E (1995) The economic impact of spinal cord injury. In: Stover SL, DeLisa JA, Whiteneck GG (eds) Spinal cord injury: clinical outcomes from the model systems. Aspen Publishers, Gaithersburg, pp 234-271

15. Salvini v. Ski Lifts, Inc. (2007) King County Superior Court, Case 05-2-13652-9 SEA, Seattle, WA, April 6, 2007

16. Bagley v. Mt. Bachelor, Inc. (2014) Supreme Court of the State of Oregon, 72 (543)

17. National Ski Areas Association (2008) Freestyle Terrain Park Notebook

18. Hubbard M (2009) Safer ski jump landing surface design limits normal velocity at impact. In: Johnson RJ, Shealy JE, Langren M (eds) Skiing trauma and safety: 17th volume, STP 1510. ASTM International, West Conshohocken, PA

19. McNeil JA, McNeil JB (2009) Dynamical analysis of winter terrain park jumps. Sports Eng 11(3):159-164

20. Bohm H, Senner V (2009) Safety in big jumps: relationship between landing shape and impact energy determined by computer simulation. In: Johnson RJ, Shealy JE, Langren M (eds) Skiing trauma and safety: 17th volume, STP 1510. ASTM International, West Conshohocken, PA

21. Swedberg AD (2010) Safer ski jumps: design of landing surfaces and clothoidal in-run transitions [MS thesis]. Naval Postgraduate School, Monterey (CA)

22. Swedberg AD, Hubbard M (2012) Modeling terrain park jumps: linear tabletop geometry may not limit equivalent fall height. In: Johnson RJ, Shealy JE, Greenwald R, Scher I (eds) Skiing trauma and safety: 19th volume, STP 1553. ASTM International, West Conshohocken, PA, pp 120-135

23. Hubbard M, Swedberg AD (2012) Design of terrain park jump landing surfaces for constant equivalent fall height is robust to "uncontrollable" factors. In: Johnson RJ, Shealy JE, Greenwald R, Scher I (eds) Skiing trauma and safety: 19th volume, STP 1553. ASTM International, West Conshohocken, PA, pp 75-94. doi:10.1520/STP104515

24. McNeil JA (2012) Modeling the 'Pop' in winter terrain park jumps. In: Johnson RJ, Shealy JE, Greenwald R, Scher I (eds) Skiing trauma and safety: 19th volume, STP 1553. ASTM International, West Conshohocken, PA, pp 95-119. doi:10.1520/STP104240

25. McNeil JA (2012) The inverting effect of curvature on winter terrain park jump takeoffs. In: Johnson RJ, Shealy JE, Greenwald R, Scher I (eds) Skiing trauma and safety: 19th volume, STP 1553. ASTM International, West Conshohocken, PA, pp 136-150. doi:10.1520/STP20120062

26. McNeil JA, Hubbard M, Swedberg AD (2012) Designing tomorrow's snow park jump. Sports Eng 15(1):1-20

27. Hubbard M, McNeil JA, Petrone N, Cognolato M (2015) Impact performance of standard tabletop and constant equivalent fall height snow park jumps. In: Johnson RJ, Shealy JE, Greenwald RM (eds) Skiing trauma and safety: 20th volume, STP 1582. ASTM International, West Conshohocken, PA, pp 51-71. doi:10. 1520/STP158220140027 
28. McNeil JA (2015) Defining and measuring freestyle snow park jumps, presentation at International Society for Skiing Safety Congress, San Vito, Italy, March 10, 2015

29. Hubbard M (2013) Luge track safety. Sports Eng 16:123-135

30. Buhl D, Fauve M, Rhyner H (2001) The kinetic friction of polyethelyn on snow: the influence of the snow temperature and the load. Cold Regions Sci Technol 33:133-140

31. Streeter VL, Wylie EB, Bedford KW (1998) Fluid mechanics. 9th ed. McGraw-Hill, Boston, pp 332-339

32. Hoerner SF (1965) Fluid dynamic drag. Bakersfield (CA): SF Hoerner (Lib. of Congress Card No. 64-19666)

33. Barelle C, Ruby A, Tavernier M (2004) Experimental model of the aerodynamic drag coefficient in alpine skiing. J Appl Biomech 20:167-176

34. Lind D, Sanders SP (1996) The physics of skiing. Springer, New York

35. Catalfamo RS (1997) Dynamic modeling of speed skiing. Am J Phys 65:1150-1156

36. Müller W, Platzer D, Schmölzer B (1995) Scientific approach to ski safety. Nature 375:455
37. US Terrain Park Council. http://usterrainparkcouncil.org. Accessed September 2011

38. Minetti AE, Ardigo LP, Susta D, Cotelli F (2010) Using leg muscles as shock absorbers: theoretical predictions and experimental results of drop landing performance. Ergonomics 41(12):1771-1791

39. International Ski Federation (2012 ) Standards for the construction of jumping hills. http://www.fis-ski.com/mm/Document/doc umentlibrary/Skijumping/03/20/22/StandardsfortheConstructio nofJumpingHills2012_english_English.pdf. Accessed November 2014

40. Schindelwig K, Reichl W, Kaps P, Mössner M, Nachbauer W (2014) Safety assessment of jumps in ski racing. Scand J Med Sci Sports. doi:10.1111/sms. 12300

41. Troy Flanagan, private communication

42. McNeil JA (2015) Designing tomorrow's jumps: engineering for safety, presentation at IOC/FIS Meeting on Injury Prevention Initiatives, Lausanne, Switzerland, June 18-19, 2015 